\title{
MARANTIC ENDOCARDITIS IN A PATIENT WITH SUSPECTED LUNG CANCER
}

Deepti Satelli MBBS ${ }^{1}$, Colleen Lechtenberg MD ${ }^{2}$

${ }^{1}$ Department of Medicine, ${ }^{2}$ Department of Neurology

UNIVERSITY OF KANSAS MEDICAL CENTER, KANSAS CITY

\section{OBJECTIVE}

To present a case of Marantic Endocarditis in a patient with suspected lung cancer.

\section{INTRODUCTION}

Marantic or Non bacterial thrombotic

endocarditis(NBTE) is a uncommon form of endocarditis usually seen in patients with underlying malignancy. Can rarely even occur in patients without malignancy.

Common manifestation is systemic embolism. Diagnosis is usually made by TEE. Treatment includes systemic anticoagulation in cases with no contraindications and work up of underlying malignancy.

\section{CASE PRESENTATION}

63 year old man presented with left arm weakness, numbness and aphasia. Imaging of his head was done. MRI showed multiple strokes in cerebral cortex. CT head did not show any evidence of hemorrhage. He was evaluated by Neurology and was started on Heparin drip. Patient had another episode of stroke after a few hours. MRI head this time showed evolution of previously noted cerebral infarcts with small areas of hemorrhage and development of new bilateral cerebral and cerebellar infarcts. Heparin drip was discontinued. Further work up was done to evaluate the etiology of thromboembolism. Transesophageal Echocardiogram(TEE) showed small vegetations on the aortic and mitral valve. $\mathrm{CT}$ scans showed splenic and bilateral renal infarcts. Patient developed peripheral stigmata of endocarditis on the next day. Blood cultures were negative, so the empiric antibiotics were discontinued. His hospital course was complicated by development of Diffuse Alveolar Hemorrhage and DIC leading to his demise. He was suspected to have lung cancer due to weight loss, hemoptysis and suspicious lesion on CT scan of chest. Further work up including diagnostic bronchoscopy could not be done due to above complications and clinical deterioration.

\section{DIAGNOSIS}

Transesophageal echocardiogram(TEE) is the preferred diagnostic modality. Lesions range from microscopic aggregates of platelets to large vegetations on heart valves, most often mitral and aortic valve. Valvular dysfunction or valvular destruction is less common. Blood cultures are negative indicating non infectious etiology. Common sites of embolization include spleen, kidney, extremities but most significant morbidity is from embolism to central nervous system. Typical stroke pattern is seen on MRI head.

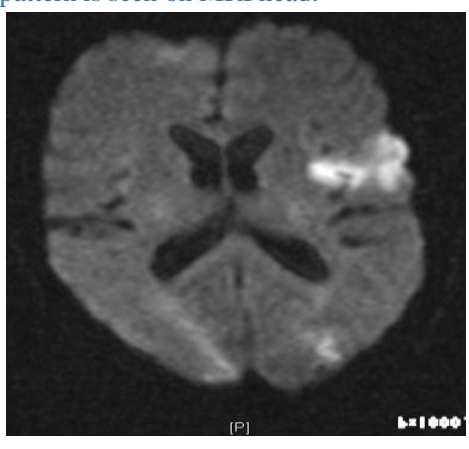

Image 1: MRI, diffusion weighted image showing typical stroke pattern.

\section{TREATMENT}

The main treatment objective is anticoagulation to prevent further episodes of thrombo embolism. Indefinite systemic anticoagulation if there are no contra indications is the main treatment. Surgical intervention is indicated in case of heart failure from valve dysfunction. Antibiotics are usually not indicated due to non infectious nature.

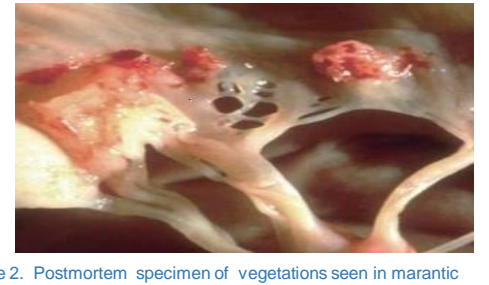

Image 2. Pestocarditis

\section{DISCUSSION}

\section{HISTORY}

Malignancy is commonly associated with

hypercoagulability. Occasionally patients may present with thrmoboembolism, marantic endocarditis.

\section{PHYSICAL EXAM}

Physical findings of systemic embolism or endocarditis are seen based on clinical course.

\section{DIAGNOSTIC TESTS}

- Blood cultures to rule out infectious endocarditis

- TEE is preferred mode to diagnose vegetations on heart valves.

- Imaging to evaluate systemic embolism. MRI especially diffusion weighted images are useful in case of stroke.

- Most importantly work up has to be performed to diagnose underlying malignancy.

\section{TREATMENT}

- Supportive care

- Anticoagulation: Indefinite systemic anticoagulation if no contraindications

- Surgical management in case of heart failure secondary to valve dysfunction.

- Treatment of underlying cause, malignancy.

\section{REFERENCES}

ism in the course of Marantic Endocarditis, Annals of thoracic surgery, Dec 2003.

Jameson G, Ramanathan R, Borad M, Downhour M, Korn R. Marantic endocarditis associated with pancreatic cancer. Case reports of Gastroenterology 2009.

Smeglin A, Ansaria M,Skali H, Oo T. Amrantic Endocarditis, DIC with systemic embol
in a patient with pancreatic cancer. American society of clinical Oncology Dec 2007 . Singhal A, Buonamno T Acute Ischemic stroke pattern in infective and non bact rial thrombotic endocarditis. Stroke 2002

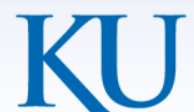

